\title{
SEARCHES FOR CATACLYSMIC VARIABLES IN GLOBULAR CLUSTER CORES
}

\author{
MICHAEL M. SHARA \\ Space Telescope Science Institute \\ 3700 San Martin Dr. \\ Baltimore, $M D 21218$ \\ USA \\ mshara@stsci.edu
}

\begin{abstract}
.
Close binaries are widely believed to exist in large numbers in the cores of globular clusters. If present, these binaries are critical sources and sinks of energy that drive the dynamical evolution of their host clusters. I report on HST searches for binaries (based on variability) in the outskirts and cores of several globular clusters; dwarf novae should be particularly easy to find. Dense and loose clusters have been thoroughly searched on timescales ranging from minutes to years. Detailed simulations demonstrate that virtually all binaries with $M<8$, amplitudes $>0.1$ mag and periods of $2-20$ hours should have been found. This includes virtually all known contact binaries. At least $1 / 3$ of all dwarf novae present in several globulars should also have been seen (very easily!) in eruption at $M=4-6$.

Simple tidal capture theory predicts that dozens of interacting binaries should have been found in our searches; the observed number is typically one or two objects per cluster. Unless tidal capture cataclysmic binaries are rapidly destroyed, ejected, or much fainter than most of their Galactic counterparts, we must conclude that very close binaries in globular cores are rare, and that their total influence on cluster dynamical evolution is less than currently claimed.
\end{abstract}

Tidal capture theory predicts that close binaries are formed by two- and three-body close encounters. The efficiency of the process (and the resulting numbers and types of interacting binaries) is currently a topic of much heated debate. The theoretical uncertainties in the physics of tidal capture 
are unlikely to be resolved soon. However, the Hubble Space Telescope has now made significant progress in observational searches for close binaries near globular cores. I briefly report on the results of three of these searches for cataclysmic binaries.

\section{NGC 6752}

The core of NGC 6752 has been imaged every 14 minutes over a span of 7 hours. 730 stars were monitored with the Hubble Space Telescope Faint Object Camera, working at $2200 \AA$ (Shara et al. 1995, ApJ, 441, 617). Artificial star tests demonstrate that we should have found virtually all variables brighter than $m_{220}=20$ (i.e., $M_{220} \leq 6.75$ ) with variability amplitude $\Delta m \geq 0.25 \mathrm{mag}$ and most variables brighter than $m_{220}=22$ (i.e., $\left.M_{220} \leq 8.75\right)$ with $\Delta m \geq 0.35 \mathrm{mag}$. No variables were found; eight were expected based on tidal capture models in the one-fourth of the core that we observed. Our results strongly constrain the number of cataclysmic and contact binaries (with the above variablility and luminosity limits) in the core of NGC 6752 to be $\leq 10$ in total with $95 \%$ probability.

\section{2. $\mathbf{M 8 0}$}

A deep $(\mathrm{U}, \mathrm{B})$ color-magnitude diagram has been obtained of the core of the dense globular M80. A well-documented classical nova was seen to erupt in M80 in May 1860. Brightening to less than 7th apparent magnitude, the nova outshone the entire cluster for a week. Its decline was followed to 10 th apparent magnitude 3 weeks later.

We have used an HST color-magnitude diagram to recover the quiescent classical nova, now known as T Sco (Shara \& Drissen 1995, ApJ, 448, 203). It is almost an order of magnitude fainter than most decades-old novae. 8000 stars were examined in the M80 CMD. Only one other object (besides $\mathrm{T}$ Sco) occupies a position (in the CMD) bluewards and faintwards of the turnoff. The presence of only one other blue, faint star in the core of M80 is striking and highly significant. Dozens of bright, blue cataclysmic variables should easily have been detectable in our HST images if simple tidal capture theory is correct.

\section{47 Tuc}

Finally, we have used archival HST images of the core of 47 Tuc (taken at 12 independent epochs) to search for erupting dwarf novae (Shara et al., 1996, submitted). We easily recovered the one known dwarf nova (V2) in 47 Tuc, and found it in eruption in 2 of our 12 epochs. 
Our spatial and temporal coverage was sufficient to locate $1 / 3$ of all dwarf novae (assuming that globular dwarf novae erupt every few weeks or months). Not a single dwarf nova (other than V2) was discovered. Our results strongly suggest that at most a few, and certainly not dozens, of cataclysmics inhabit the core of 47 Tuc. The one "escape hatch" that may still permit many interacting binaries to exist in globular cores is brightness - or lack thereof. If mass transfer rates are low (e.g. if most catalysmics are strongly magnetic or in hibernation most of the time) then simple tidal capture theory may still be vindicated.

\section{Conclusions}

Before HST resolved the cores of many Galactic globular clusters, theorists had predicted a plethora of cataclysmic variables in their cores. While a few CVs have now been found, the numbers appear to be one to two orders of magnitude less than the early expectations. Deeper searches with HST will soon show if tidal capture binaries, and in particular CVs, are truly rare, or if most CVs are intrinsically very faint. 